\title{
Antagonistic Role of Aqueous Complexation in the Solvent Extrac- tion and Separation of Rare Earth lons
}

\author{
Pan Sun ${ }^{1}$, Erik A. Binter ${ }^{2}$, Zhu Liang ${ }^{2 \dagger}$, M. Alex Brown ${ }^{3}$, Artem V. Gelis ${ }^{4}$, Ilan Benjamin ${ }^{5}$, Mrinal K. \\ Bera1, Binhua Lin¹, Wei Bu1*, Mark L. Schlossman²* \\ ${ }^{1}$ NSF's ChemMatCARS, Pritzker School of Molecular Engineering, University of Chicago, Chicago, IL 60637, USA. \\ 2Department of Physics, University of Illinois at Chicago, Chicago, IL 60607, USA. \\ ${ }^{3}$ Chemical and Fuel Cycle Technologies Division, Argonne National Laboratory, Lemont, IL, 60439, USA. \\ ${ }^{4}$ Radiochemistry Program, Department of Chemistry and Biochemistry, University of Nevada, Las Vegas, NV, 89141, \\ USA. \\ ${ }^{5}$ Department of Chemistry and Biochemistry, University of California, Santa Cruz, California 95064, USA
}

\begin{abstract}
During solvent extraction of rare earth ions, an aqueous electrolyte solution is placed in contact with an immiscible organic solution of extractants to enable extractant-facilitated transport of ions into the organic solvent. Although experimental methodologies such as x-ray and neutron scattering have been applied to characterize ion-extractant complexes, identifying the site of ion-extractant complexation has proven challenging. Here, we use tensiometry and surface-sensitive $x$ ray scattering to study the surface of aqueous solutions of lanthanide chlorides and the water-soluble extractant bis(2ethylhexyl) phosphate (HDEHP), in the absence of a coexisting organic solvent. These studies restrict interactions of HDEHP with trivalent lanthanide ions to the aqueous phase and the liquid-vapor interface, allowing us to explore the consequences that one or the other is the site of ion-extractant complexation. Unexpectedly, we find that light lanthanides preferentially occupy the liquid-vapor interface, with an overwhelming preference for a light lanthanide, $\mathrm{Nd}$, when present in a mixture with a heavy lanthanide, Er. This contradicts our expectation that heavy lanthanides should have a higher interfacial density since they are preferentially extracted by HDEHP in the presence of an organic phase. These results reveal the antagonistic role played by ion-extractant complexation within the aqueous phase and clarify the potential advantages of water-insoluble extractants that interact with ions primarily at the interface during the process of solvent extraction.
\end{abstract}

\section{INTRODUCTION}

Extractant-assisted transport of rare earth metal ions across the liquid-liquid interface between immiscible aqueous and organic phases underlies their separation and purification by solvent (i.e., liquid-liquid) extraction processes.-2 In spite of the importance of rare earth ions to a diverse range of critical and evolving technologies, ${ }^{3-5}$ the separation mechanism on the nanoscale is not well understood. This mechanism relies upon the interaction and complexation of metal ions with organic extractants in the neighborhood of a liquid interface.

Early studies of the solvent extraction of the lanthanide (Ln) elements, which make up most of the rare earths, demonstrated the utility of organophosphoric acid extractants such as bis(2-ethylhexyl) phosphate (HDEHP) dissolved in organic solvents. ${ }^{6}$ When the organic phase is placed in contact with an immiscible aqueous phase of lanthanides, HDEHP complexes with lanthanide ions and extracts them into the bulk organic phase. This extraction can be more efficient for some lanthanides than for others, leading to its use to separate and purify individual lanthanide elements. Generally, lanthanides are divided into two groups, based upon their position in the lanthanide (4-f element) series: light and heavy lanthanides. The separation of lanthanides from different groups is relatively easy compared with those located within the same group. For example, the separation factor between the light lanthanide neodymium, Nd, and the heavy lanthanide erbium, Er can reach about 100, while the separation factor between neighboring lanthanides is usually between 1 and $2 .{ }^{7}$ As a result, separation of neighboring lanthanides often involves repeated cycling of the extraction process, complicating the process of separations and increasing the cost of production and the risk of environmental pollution..$^{8-9}$ Although a great effort has been made during the past 50 years to improve the separation of lanthanides by optimizing the extraction process conditions and synthesizing new extractants, there is still a need to better understand the extraction mechanism on the molecular scale in order to optimize the separation of neighboring lanthanides or even those within a single group. 
The weak amphiphilic nature of many extractants suggests that the formation of metal ion-extractant complexes occurs preferentially at the organic-aqueous interface. ${ }^{10-13}$ Many extractants contain a hydrophilic, polar headgroup responsible for interacting with metal ions in the aqueous phase and a hydrophobic tailgroup that provides solubility in the organic phase. Interfacial tension measurements demonstrate significant interfacial activity of extractants, which tend to accumulate at the organic-aqueous interface, where their encounter with metal ions is likely to occur. ${ }^{11,14}$ However, investigations into the kinetics of lanthanide ion complexation and transport have suggested that extractants can bind to ions either at the interface (Path 1 in Fig. 1) or in the region of the aqueous phase boundary layer near the interface if the extractants are also water-soluble, as is often the case (Path 2 in Fig. 1). The mass transfer with chemical reaction (MTWCR) mechanism includes the possibility illustrated by Path 2 that water-soluble acidic extractants, such as HDEHP, are transferred into the aqueous boundary layer near the organic-aqueous interface, where they are deprotonated and interact with metal ions to form aqueous ion-extractant complexes, which subsequently diffuse into the organic phase. ${ }^{15-}$ 17 An example includes the extraction of Zn(II) with diphenylthiocarbazone (dithizone) for which kinetics studies suggested complexation in the bulk aqueous phase as in Path $2 .{ }^{18}$ Subsequent kinetics studies of more hydrophobic variants of dithizone suggested that complexation occurs at the interface as in Path $1 .{ }^{10}$ The relative advantage of ion-extractant complexation occurring either at the interface or in the aqueous phase is not well explored and is addressed here in the context of HDEHP extraction of trivalent lanthanides.

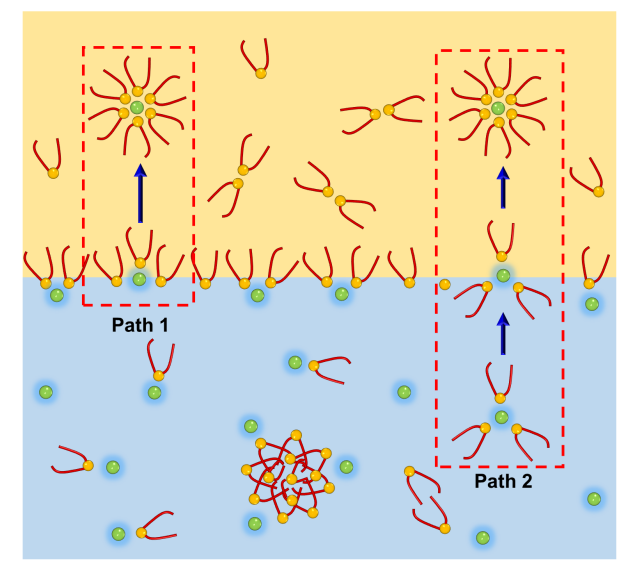

Figure 1. Two sites of ion-extractant complexation define the initial state of two different paths for transport of ions across the liquidliquid interface during solvent extraction. Path 1 starts at the interface as the site of initial ion-extractant complexation. Path 2 starts in the aqueous phase boundary layer as the site of initial complexation.

Interface-sensitive experimental methods have begun to probe the structure of interfacial ion-extractant complexes and the role of hydrogen bonding in their formation and stabilization at the aqueous-organic interface. ${ }^{19-25}$ For instance, a thermal switch was developed to arrest interfacial complexes of lanthanides and water-insoluble extractants formed in the midst of the extraction process, thereby allowing for the characterization of intermediate interfacial states by $\mathrm{x}$-ray scattering. ${ }^{21-22}$ Model systems of water-insoluble surfactant monolayers at the liquid-vapor interface have also been studied by interface-sensitive methods to explore the interactions between ions and surfactants whose headgroups mimic those of more weakly amphiphilic extractants. ${ }^{26-37}$ Although studies at the liquid-vapor interface cannot model ion transport through the interface or structures that may form uniquely at a liquid-liquid interface, they may be able to model many aspects of the nanoscale variation of density, dielectric properties, and water structuring near a liquid interface that can influence ion-extractant complexation. However, studies of water-insoluble surfactants or extractants cannot address the issue of the site of complexation of water-soluble extractants.

Recently, Kusaka and Watanabe studied the liquid-vapor interface of aqueous solutions containing water-soluble HDEHP and lanthanide ions.38-39 They used vibrational sum frequency generation spectroscopy measurements to suggest the coordination of ion-extractant complexes at the interface. Here, we study the liquid-vapor interface of aqueous solutions containing lanthanide chlorides and water-soluble HDEHP with tensiometry, x-ray reflectivity and x-ray fluorescence. By omitting the bulk organic phase, these studies restrict interactions of HDEHP with trivalent lanthanide ions to the bulk aqueous phase, the aqueous boundary layer near the interface, and the liquid-vapor interface. This allows us to explore the consequences of ion-extractant complexation occurring in either the aqueous phase or at the interface. Unexpectedly, we find that light lanthanides preferentially occupy the water-vapor interface. This contradicts our expectation that heavy lanthanides should have a higher interfacial density since they are preferentially extracted by HDEHP in the presence of an organic phase. ${ }^{6-7}$ We also examined the complexation between Ln ions and a monolayer of insoluble di-hexadecyl phosphate (DHDP), which has the same phosphoric acid headgroup as HDEHP but is confined to the aqueous liquid- 
vapor interface, and found an enhanced presence of heavy over light Ln ions, as expected. Comparison of these results reveals the antagonistic role played by ion-extractant complexation within the aqueous phase.

\section{EXPERIMENTAL SECTION}

Materials. Ultrapure water from a Millipore system with resistivity of $18.2 \mathrm{M} \Omega \bullet \mathrm{cm}$ was used for all aqueous solutions. Bis(2-ethylhexyl)-phosphoric acid (HDEHP, Chart 1) was purchased from Alfa-Aesar (97\%) and purified to >99.9\% via a third-phase formation procedure. $\mathrm{NdCl}_{3} \bullet 6 \mathrm{H}_{2} \mathrm{O}(99.9 \%)$ and $\mathrm{ErCl}_{3} \bullet 6 \mathrm{H}_{2} \mathrm{O}(99.995 \%) \mathrm{LuCl}_{3} \bullet 6 \mathrm{H}_{2} \mathrm{O}(99.99 \%), \mathrm{GdCl}_{3} \bullet 6 \mathrm{H}_{2} \mathrm{O}$ (99.99\%), DyCl $\bullet_{3} 6 \mathrm{H}_{2} \mathrm{O}$ (99.9\%), were purchased from Sigma Aldrich and used without further purification. $\mathrm{LaCl}_{3} \bullet 7 \mathrm{H}_{2} \mathrm{O}$ (99.99\%) was purchased from Alfa-Aesar and used without further purification. Dihexadecyl phosphate (DHDP, >98\% purity from Sigma-Aldrich, Chart 1) was purified by recrystallizing it twice from chloroform.

\section{Chart 1. Molecular Structure of (A) HDEHP and (B) DHDP}

A

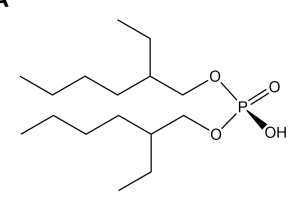

B

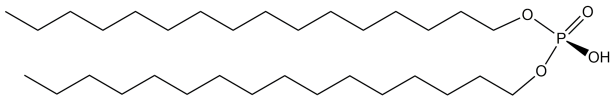

Solutions. Aqueous solutions of bis(2-ethylhexyl) phosphate (HDEHP, Chart 1) and lanthanide chlorides $\left(\mathrm{LnCl}_{3}\right.$ ) were prepared as described in SI1.2. Aqueous solutions were prepared at three different values of $\mathrm{pH}: 2.0,3.0$, and 4.5. The percentages of protonated HDEHP and deprotonated $\mathrm{DEHP}^{-}$in a bulk aqueous solution without lanthanides were calculated for these $\mathrm{pH}$ values by using a pKa of 3.24:40 5\% DEHP $^{-}$and 95\% HDEHP at pH 2.0, 37\% DEHP $^{-}$and $63 \%$ HDEHP at pH 3.0, and 95\% DEHP ${ }^{-}$and 5\% HDEHP at pH 4.5 (Fig. S1). Note that the presence of lanthanides can change these percentages as the proton and the Ln(III) compete for the DEHP-. The pH values used in these measurements span a range for which the phosphoric acid extractant is mostly protonated (HDEHP) at $\mathrm{pH} 2.0$, mostly deprotonated (DEHP ${ }^{-}$) at $\mathrm{pH}$ 4.5 , and is present in intermediate quantities of both protonated and deprotonated species at $\mathrm{pH} 3.0$. These $\mathrm{pH}$ values cover the range of acidities that are typically used in solvent extraction of lanthanides by HDEHP. Note that at values of $\mathrm{pH}$ below 2.0, the complexation between Ln and HDEHP is expected to be insignificant. We will use the shorthand notation (H)DEHP in contexts that do not distinguish between DEHP ${ }^{-}$and HDEHP species.

Aqueous solutions were prepared with water-soluble (H)DEHP at a 6:1 number ratio of (H)DEHP:Ln for each lanthanide in solution (SI). The ratio was chosen based upon known values of (H)DEHP complexation with lanthanides in bulk organic phases, but do not necessarily represent the complexation ratio for the bulk aqueous phases studied here. ${ }^{7}$ The (H)DEHP concentration in the bulk aqueous solution for all samples was $10 \pm 1 \mu \mathrm{M}$, as measured by ICP-OES, which is a factor of 30 smaller than the saturation concentration measured at $\mathrm{pH} 3.4$.

Considerations of ion-extractant complexation discussed in this paper will be affected by the values of the critical micelle concentration (CMC) for HDEHP, which we measured to be $1.6 \mu \mathrm{M}$ (Fig. S2) in the absence of Ln ions, and for DEHP-, which has been reported to be $18 \mathrm{mM}$ in the absence of Ln ions. ${ }^{41}$ Therefore, the concentration of HDEHP in all of our samples is above its Ln-ion-free $\mathrm{CMC}$ for $\mathrm{pH} 2.0$ and $\mathrm{pH} 3.0$ samples, but below its $\mathrm{Ln}$-ion-free $\mathrm{CMC}$ for the $\mathrm{pH} 4.5$ sample, and the concentration of DEHP- in all of our samples is below its Ln-ion-free CMC. Aqueous solutions containing $10 \pm 1 \mu \mathrm{M}$ (H)DEHP were also prepared without Ln ions, referred to in the figures below as "no Ln ions". All measurements were taken at room temperature $\left(22^{\circ} \mathrm{C}\right)$.

Methods. The liquid-vapor interfaces of aqueous solutions were studied with tensiometry (SI 1.3), x-ray reflectivity (SI 1.5), and $\mathrm{x}$-ray fluorescence near total reflection (XFNTR) (SI 1.6). X-ray measurements used $10 \mathrm{keV} x$-rays with the liquid surface scattering instrument at Sector 15 (SI 1.4), ${ }^{42-43}$ NSF's ChemMatCARS, of the Advanced Photon Source at Argonne National Laboratory. See the SI for a detailed description of experimental and MD simulation methods. 


\section{RESULTS}

3.1 Surface Tension Measurements. Figure 2 presents surface tension measurements from the liquid-vapor interface of aqueous solutions of (H)DEHP and a series of lanthanide chlorides for three values of $\mathrm{pH}(2.0,3.0$, and 4.5). These curves illustrate the variation of surface tension with time as well as the equilibrium value attained after measuring for a sufficiently long time. In the absence of lanthanide ions, aqueous solutions of (H)DEHP have an equilibrium value of surface tension that decreases as the $\mathrm{pH}$ is lowered. Aqueous solutions containing $(\mathrm{H}) \mathrm{DEHP}$ and lanthanide chlorides at $\mathrm{pH}$ 2.0,3.0, or 4.5 produce an equilibrium surface tension that is highest for solutions containing the heaviest lanthanide ions ( $\mathrm{Lu}$ ) and sequentially smaller for solutions containing lighter lanthanide ions (from Lu to Er, Dy, Gd, Nd, to La). Xray measurements presented later will confirm the interpretation of these surface tension measurements that surface adsorption is greater in the presence of lighter lanthanides.

Figure 2 also illustrates the kinetics of attaining equilibrium. It is generally observed that solutions with lighter lanthanides equilibrate quickly, more or less at the rate with which (H)DEHP equilibrates in the absence of Ln ions, but the kinetics is slower for solutions with heavier lanthanides. Similar Ln extraction kinetics has been reported for the TALSPEAK system: 1M HDEHP/0.05M DTPA in a lactate buffer ${ }^{44-45}$ The slow equilibration is particularly apparent at $\mathrm{pH}$ 4.5 where it can be observed that the surface tensions for the single-Ln-ion component samples containing Er and Lu ions persist at values near that of pure water for 100 minutes or more, suggesting that these ions stabilize an interface that has little extractant even on time scales much longer than required for the equilibration of (H)DEHP in the absence of Ln ions. Eventually, the surface tension relaxes to values lower than that of pure water, e.g., $66.6 \mathrm{mN} / \mathrm{m}$ for Er ions, indicating a slow adsorption to the liquid-vapor interface, an adsorption that is confirmed by x-ray measurements, as discussed later. On the other hand, the surface tension of solutions with lighter lanthanides, for example Nd ions, relaxes on the same time scale as in the absence of ions, suggesting that the relaxation is dominated by the adsorption kinetics of (H)DEHP to the interface. Similar effects are observed to a lesser extent at pH 2.0 and 3.0.
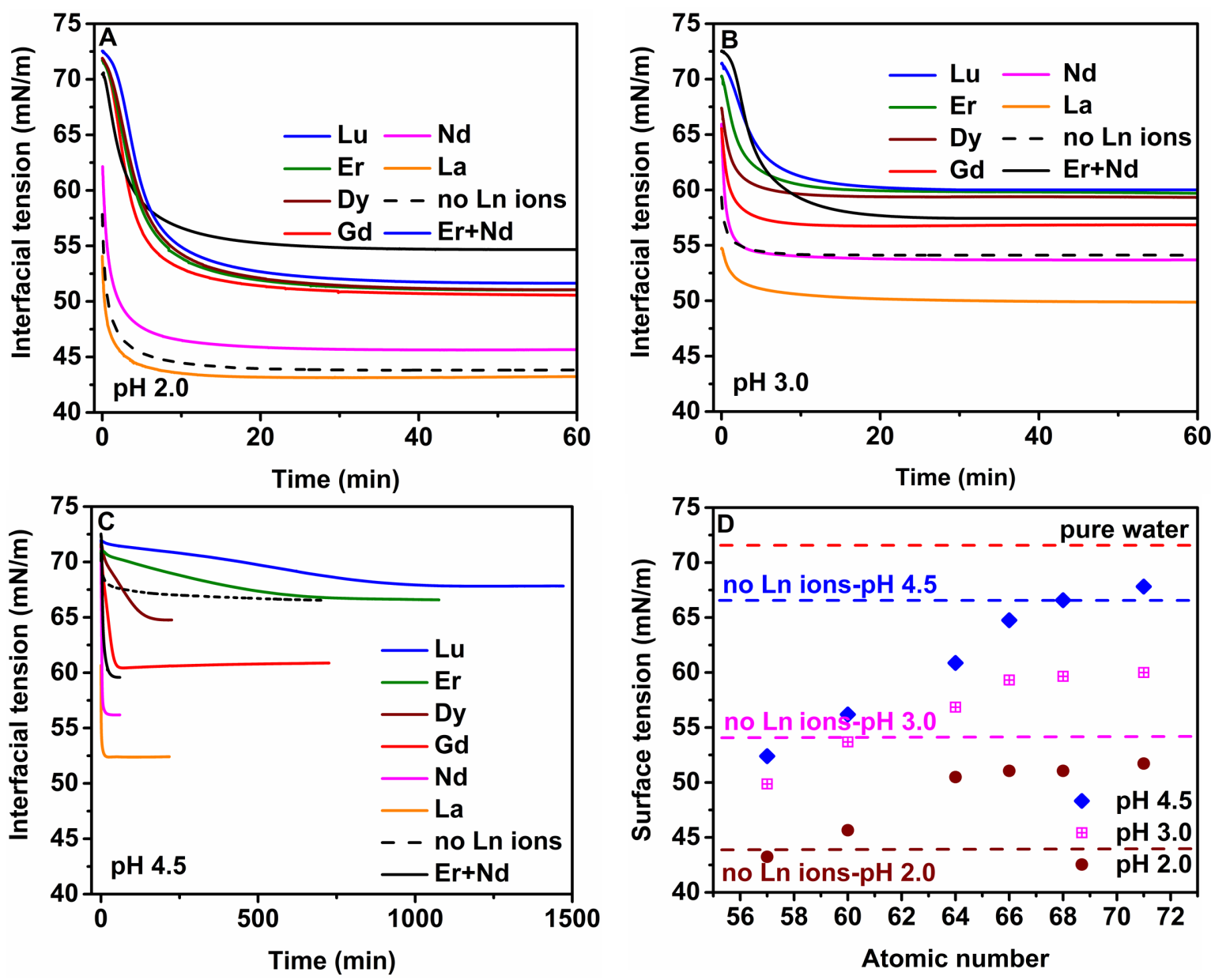

Figure 2. Surface Tension Measurements. Relaxation and equilibrium values of the liquid-vapor surface tension of (H)DEHP-aqueous solutions in the absence ("no Ln ions") or presence of lanthanide chlorides. (A) $\mathrm{pH}$ 2.0, (B) pH 3.0, (C) pH 4.5, (D) equilibrium values of the surface tension vs. atomic number [La(57), $\mathrm{Nd}(60), \mathrm{Gd}(64), \mathrm{Dy}(66), \mathrm{Er}(68), \mathrm{Lu}(71)$ ] for different values of pH, where dashed lines indicate the values in the absence of lanthanide ions (Table S1 lists the values). Note that the total ionic strength of the mixtures $(\mathrm{Er}+\mathrm{Nd})$ in panels A-C is twice that of the single ionic component solutions. 
3.2 Electron Density Profiles of the Surface Layer X-ray reflectivity measures the electron density profile with subnanometer resolution, where the profile is the variation in electron density along the interfacial normal but averaged over the $\mathrm{x}$-ray footprint in the plane of the liquid-vapor interface. ${ }^{46}$ Since the interfacial region may consist of different components - water, (H)DEHP, and lanthanide ions - each with a different electron density, $\mathrm{x}$-ray reflectivity can be sensitive to the arrangement of these components at the interface. However, $\mathrm{x}$-ray reflectivity cannot distinguish between HDEHP and DEHP- . Element specific information that distinguishes one lanthanide from another will be measured by XFNTR, discussed in the next section.

We have limited our x-ray studies of these systems to aqueous solutions containing (H)DEHP without Ln ions, (H)DEHP with a representative heavy lanthanide, $\mathrm{Er}(\mathrm{III})$, and a representative light lanthanide, $\mathrm{Nd}(\mathrm{III})$, as well as (H)DEHP with a 1:1 mixture of these two lanthanides. Figure 3 illustrates x-ray reflectivity measurements from the liquid-vapor interface of these aqueous samples whose composition and $\mathrm{pH}$ are identical to those measured with tensiometry. The lowest curves shown in panels $\mathrm{A}, \mathrm{B}$, and $\mathrm{C}$ for each $\mathrm{pH}$ illustrate the measurement of aqueous solutions containing water-soluble (H)DEHP without Ln ions. Other curves in panel A for $\mathrm{pH} 2.0$ samples with Ln ions are not greatly different. A broad peak develops at $Q_{z} \approx 0.2 \AA^{-1}$ for most of the samples with Ln ions at pH 3.0 and 4.5 except for those with only Er ions at pH 3.0.
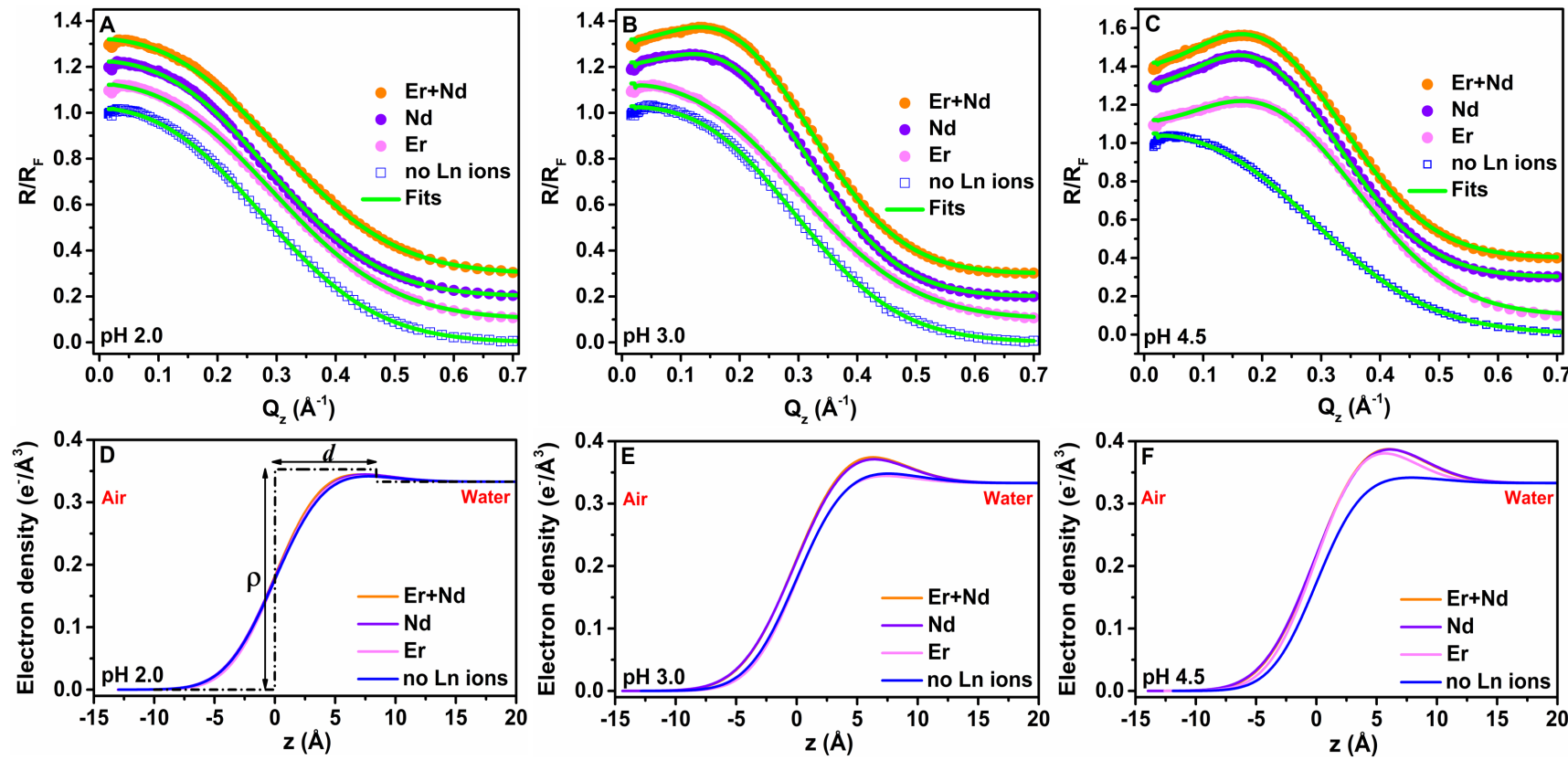

Figure 3. X-ray Reflectivity Measurements and Analysis. (A-C) X-ray reflectivity $R$ normalized to the Fresnel reflectivity $R_{F}$ as a function of wave vector transfer $Q_{z}$ normal to the surface. Measurements are from the liquid-vapor interface of equilibrated aqueous samples for three different values of pH: pH 2.0 in panel (A), pH 3.0 in (B), and pH 4.5 in (C). The samples contained (H)DEHP, ErCl3, and $\mathrm{NdCl}_{3}$ at the same concentrations used for the surface tension measurements shown in Fig. 2. The label "Er+Nd" indicates a 1:1 mixture of $\mathrm{ErCl}_{3}$ and $\mathrm{NdCl}_{3}$; "no Ln ions" refers to aqueous solutions containing (H)DEHP, but without lanthanide ions. Data are displaced for visual clarity with the vertical placement of data sets corresponding to the ordering in the legend. Note that all measured reflectivity curves approach 1 as $Q_{z}$ approaches 0 . Lines represent the best fits of the reflectivity data. (D-F) Electron density profiles as a function of the distance $z$ normal to the liquid-vapor interface. The vapor above the interface occurs at $z<<0$ and the bulk aqueous phase at $z>>0$. The dash-dot line in panel D displays a zero-roughness profile to illustrate the thickness $d$ and electron density $\rho$ of the interfacial slab. The profiles are the result of the fits shown in panels A, B, and C, respectively.

All reflectivity curves are fit with a simple model of the electron density profile that consists of a single homogeneous slab of thickness $d$ and electron density $\rho$ on top of an aqueous bulk phase represented by a homogeneous electron density, as illustrated by the dash-dot line in Fig. 3D. This profile is then convoluted with a gaussian function to account for capillary wave fluctuations of the interface. ${ }^{46}$ Note that the slab illustrated by the dash-dot line in Fig. 3D is drawn in the absence of capillary wave roughening (see Fig. S3 for zero-roughness profiles for all data sets). The interface slab represents a layer composed of (H)DEHP, water, and lanthanide ions. Panels D, E, and F of Fig. 3 illustrate the electron density profiles that result from fits to the data in panels $A, B$, and C. The lines in panels $D, E$, and F indicate the values of the electron density in the vapor and bulk aqueous phases, 0 and $0.333 \mathrm{e}^{-} / \AA^{3}$ at large negative and positive $z$, respectively, as well as the gradual crossover from the water-saturated helium vapor above the solution through the aqueous interfacial layer to the aqueous bulk phase. The gradual crossover is primarily the result of thermal capillary waves that roughen the surface. The electron density in the interfacial region rises above the value of the bulk aqueous phase. This additional electron density is the result of adsorption of $(\mathrm{H}) \mathrm{DEHP}$ and ions to the surface of the aqueous solution. It is apparent that 
the development of a broad peak at $Q_{z} \approx 0.2 \AA^{-1}$ for most of the samples with Ln ions at $\mathrm{pH} 3$ and pH 4.5 is the result of the enhanced electron density at the interface shown in panels $\mathrm{E}$ and $\mathrm{F}$.

The slab that represents the interfacial layer is described by three parameters: the layer thickness $d$, the layer electron density $\rho$, and the interfacial roughness $\sigma$. Table 1 lists the values of these parameters determined by fits to the x-ray reflectivity data. The interfacial layer is thin, with a thickness that varies from 7 to $9 \AA$. The resolution of these $x$-ray reflectivity measurements is inadequate to distinguish the location of the lanthanide ions separately from the location of (H)DEHP within this thin interfacial layer. The electron density of the interfacial layer is $4 \%$ to $30 \%$ larger than the electron density of pure water, depending upon the sample composition. The interfacial roughness varies from 3 to $3.6 \AA$, and is primarily due to capillary wave fluctuations of the interface. 46

As expected from the nearly identical reflectivity curves and corresponding electron density profiles measured for $\mathrm{pH}$ 2.0 samples, the parameters in Table 1 that describe the profiles of these samples are the same within experimental uncertainty. Samples containing Nd ions at pH 3.0 and all samples with $\mathrm{Ln}$ ions at $\mathrm{pH} 4.5$ form thinner layers than in the absence of ions, but with higher electron density. This suggests that Ln ions alter the (H)DEHP conformation at the surface. Note, however, that the thinning of the layer is not observable in Fig. 3E/F because the larger roughness for these samples smears the profile in the $z$-direction. The electron density $\rho$ is significantly larger for Nd-containing samples at pH 3.0 and for both $\mathrm{Er}$ and Nd-containing samples at pH 4.5, indicating substantial ion density at the liquid-vapor interface which will be verified by XFNTR in the next section.

Table 1. Fitting parameters from $\mathrm{X}$-ray reflectivity measurements.

\begin{tabular}{ccccc}
\hline $\mathrm{pH}$ & Ions & $d(\AA)$ & $\rho\left(\mathrm{e}^{-} / \AA^{3}\right)$ & $\sigma(\AA)$ \\
\hline \multirow{4}{*}{2.0} & None & $8.4_{-1.1}^{+0.7}$ & $0.353_{-0.003}^{+0.008}$ & $3.2_{-0.1}^{+0.2}$ \\
& $\mathrm{Er}$ & $8.3_{-1.3}^{+0.8}$ & $0.351_{-0.003}^{+0.009}$ & $3.1_{-0.2}^{+0.1}$ \\
& $\mathrm{Nd}$ & $8.1_{-1.2}^{+0.4}$ & $0.36_{-0.01}^{+0.03}$ & $3.3_{-0.2}^{+0.1}$ \\
& $\mathrm{Nd}+\mathrm{Er}$ & $7.1_{-0.8}^{+1.6}$ & $0.364_{-0.003}^{+0.09}$ & $3.1_{-0.2}^{+0.1}$ \\
\hline \multirow{4}{*}{3.0} & $\mathrm{None}$ & $9.3_{-0.6}^{+0.4}$ & $0.359_{-0.003}^{+0.005}$ & $3.2_{-0.2}^{+0.1}$ \\
& $\mathrm{Er}$ & $8.8_{-0.6}^{+0.4}$ & $0.354_{-0.003}^{+0.002}$ & $3.0_{-0.1}^{+0.1}$ \\
& $\mathrm{Nd}$ & $7.3_{-0.9}^{+0.6}$ & $0.42_{-0.02}^{+0.02}$ & $3.6_{-0.1}^{+0.1}$ \\
& $\mathrm{Nd}+\mathrm{Er}$ & $7.4_{-0.7}^{+0.6}$ & $0.43_{-0.01}^{+0.02}$ & $3.5_{-0.1}^{+0.2}$ \\
\hline \multirow{4}{*}{4.5} & $\mathrm{None}$ & $9.9_{-0.5}^{+0.4}$ & $0.346_{-0.001}^{+0.001}$ & $3.0_{-0.1}^{+0.1}$ \\
& $\mathrm{Er}$ & $7.1_{-0.6}^{+0.8}$ & $0.43_{-0.01}^{+0.02}$ & $3.2_{-0.1}^{+0.2}$ \\
& $\mathrm{Nd}$ & $7.7_{-0.8}^{+0.6}$ & $0.44_{-0.02}^{+0.02}$ & $3.5_{-0.1}^{+0.2}$ \\
& $\mathrm{Nd}+\mathrm{Er}$ & $7.7_{-0.8}^{+0.6}$ & $0.44_{-0.01}^{+0.02}$ & $3.4_{-0.1}^{+0.1}$ \\
\hline
\end{tabular}

Parameters: layer thickness $d$; layer electron density $\rho$; interfacial roughness $\sigma$.

Uncertainties on the parameters are determined by mapping the chi-squared space. ${ }^{47}$

3.3 Ion-specific Interfacial Density Although x-ray reflectivity measures the electron density profile resulting from all species at the liquid-vapor interface of the aqueous solution, it does not identify the presence of specific elements. Therefore, $\mathrm{x}$-ray fluorescence near total reflection (XFNTR) was used to measure the element-specific interfacial density in units of number of ions per area (see SI) for the same samples studied by x-ray reflectivity. Panel A in Figure 4 illustrates the increase in XFNTR signal with $\mathrm{pH}$ for equilibrated samples containing either $\mathrm{Nd}$ or Er ions. Analysis of these signals relies upon the measurement of a scale factor that differs for $\mathrm{Nd}$ and $\mathrm{Er}$, such that the same interfacial density of fluorescing ions produces a larger signal for Er than for Nd (Fig. S5). Analysis of these data is summarized in Fig. 4C, which demonstrates the increase in the interfacial density of ions as the $\mathrm{pH}$ increases from 2.0 to 4.5. More Nd ions are adsorbed to the interface than Er ions at each value of $\mathrm{pH}$. Table 2 displays values of the interfacial area per ion, just the reciprocal of the ion interfacial density shown in Fig. 4C. 
Aqueous samples of $(\mathrm{H})$ DEHP that contain a 1:1 mixture of $\mathrm{NdCl}_{3}$ and $\mathrm{ErCl}_{3}$, both in a ratio of 6:1 (H)DEHP:ion, demonstrate the result of competitive adsorption between these Ln ions. Under these conditions, Fig. 4B/D and Table 2 demonstrate that the density of Er ions at the interface drops to very low levels as $\mathrm{Nd}$ dominates the surface adsorption at all values of $\mathrm{pH}$. The surface adsorption of Nd ions in mixtures with Er ions is similar to that in the absence of Er ions.
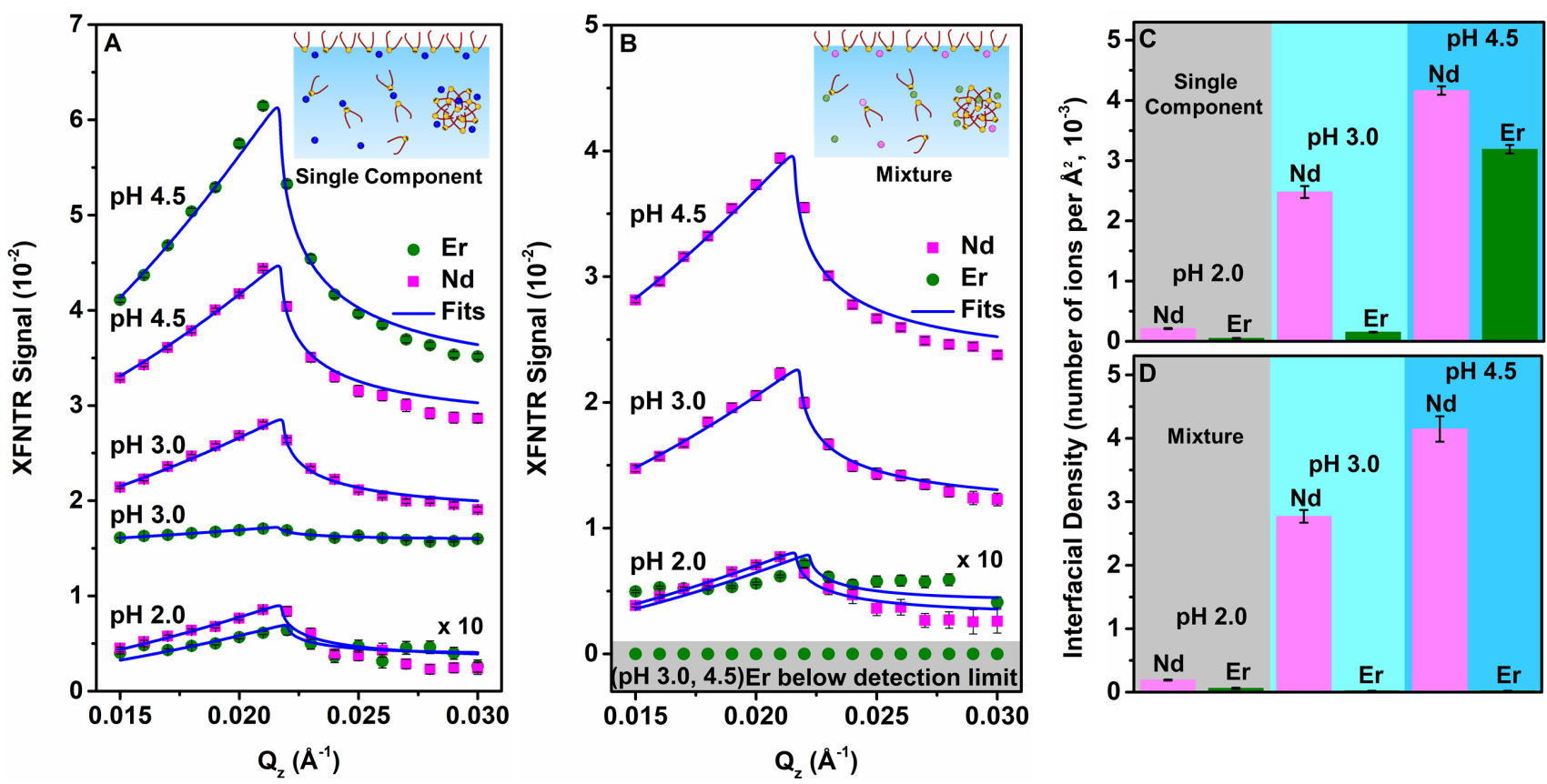

Figure 4. X-ray fluorescence near total reflection (XFNTR) as a function of the wave vector transfer $Q_{z}$ near the critical $Q_{c} \approx$ $0.217 \AA^{-1}$ for total reflection. Measured sample concentrations are the same as those in Fig. 3. (A\&C) Measurements from

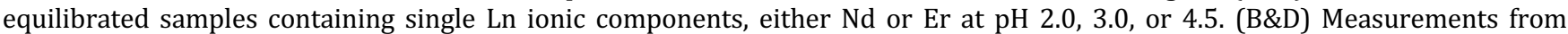
equilibrated samples containing a mixture of $\mathrm{Nd}$ and Er ions, each at a 6:1 ratio of (H)DEHP:ion, at pH 2.0, 3.0, or 4.5. (A\&B) The XFNTR signal axis scale is the same for all samples, though the pH 2.0 data have been multiplied by a factor of 10 . (C\&D) Interfacial density of ions (number per area) at different pH (Table S2). Note that the interfacial density scales with XFNTR signal in panels A and B differently for $\mathrm{Nd}$ and Er because the X-ray fluorescence yield is different for the two elements (Fig. S5). Insets to panels A and $B$ indicate that ions and extractant (H)DEHP can be either at the interface or in the bulk aqueous phase, though XFNTR detects ions at the interface.

Table 2. Interfacial Area per Ion (in $\AA^{2}$ ) of Nd and Er measured by XFNTR.

\begin{tabular}{ccccc}
\hline & \multicolumn{2}{c}{ Single Ion Samples } & \multicolumn{2}{c}{ Mixture of Ions } \\
$\mathrm{pH}$ & $\mathrm{Nd}$ & $\mathrm{Er}$ & $\mathrm{Nd}$ & $\mathrm{Er}$ \\
\hline 2.0 & $4,700(200) \AA^{2}$ & $19,900(1600)$ & $5,300(200)$ & $16,000(3,000)$ \\
3.0 & $400(20)$ & $6500(300)$ & $360(20)$ & $\mathrm{BDL}$ \\
4.5 & $240(10)$ & $310(10)$ & $240(10)$ & $\mathrm{BDL}$ \\
\hline
\end{tabular}

BDL - below detection limit $\left(\gtrsim 50,000 \AA^{2}\right)$

Estimated standard deviations at the one-sigma level are shown in parentheses and were determined by mapping the chi-squared space. 47

\section{DISCUSSION}

These studies of trivalent lanthanide ion adsorption to the liquid-vapor interface of aqueous electrolyte solutions containing the water-soluble extractant $(\mathrm{H})$ DEHP demonstrate the preferential adsorption of light over heavy lanthanides. Measurements were presented from three kinds of solutions: (1) single-Ln-component solutions with (H)DEHP, (2) solutions of two lanthanides ( $\mathrm{Nd}$ and Er) with (H)DEHP, and (3) solutions of (H)DEHP without lanthanides. It is worthwhile noting that surface tension and $\mathrm{x}$-ray measurements of aqueous solutions of lanthanide chlorides at the micromolar concentrations used in these experiments, but in the absence of $(\mathrm{H})$ DEHP, are essentially indistinguishable from measurements of the surface of pure water. 
Preferential Adsorption of Lighter Lanthanides at the Surface of Water-Soluble HDEHP Solutions. Figure 2 showed that the surface tensions of single Ln-component solutions become successively lower with decreasing lanthanide mass from Lu to Er, Dy, Gd, Nd, to La for each pH studied (2.0, 3.0, and 4.5). X-ray measurements in Figures 3 and 4 compared the behavior of aqueous (H)DEHP solutions with $\mathrm{Nd}$ ions, a light lanthanide, to solutions with Er ions, a heavy lanthanide. The enhanced electron densities measured by X-ray reflectivity occur primarily at higher $\mathrm{pH}$ (3.0 and 4.5) in the presence of the lighter Nd ions, though a significant, but slightly lower electron density is also observed for the heavier Er ions at the highest $\mathrm{pH}$ of 4.5 (Fig. 3). X-ray fluorescence measurements (XFNTR) demonstrate that the interfacial density of Nd ions is larger than that of Er ions at the liquid-vapor interface of single Ln-component solutions for all $\mathrm{pH}$ values studied (Fig. 4C). Studies of solutions containing both $\mathrm{Nd}$ and Er ions reveal an overwhelming preference for ions of the lighter lanthanide, $\mathrm{Nd}$, at the liquid-vapor interface, again, for all $\mathrm{pH}$ values studied (Fig. 4D).

pH Dependence in the Absence of Ln Ions. Varying pH has a substantial impact on the results, most likely due to the varying degree of protonation of $(\mathrm{H}) \mathrm{DEHP}$ in the bulk aqueous phase. The fraction of protonated species, HDEHP, can be calculated, in the absence of $\mathrm{Ln}$ ions, to vary from $95 \%$ at pH 2.0 to $63 \%$ at pH 3.0 to $5 \%$ at pH 4.5 (Fig. S1). This variation influences the surface tension even in the absence of Ln ions. At $\mathrm{pH} 4.5$, without Ln ions, the concentration of both HDEHP and DEHP- are below their respective values of critical micelle concentration and the surface tension is reduced to 66.5 $\mathrm{mN} / \mathrm{m}$, only slightly below that of pure water at $72 \mathrm{mN} / \mathrm{m}$, indicative of a small adsorption of (H)DEHP to the interface. At $\mathrm{pH}$ 3.0, without lanthanide ions, the concentration of HDEHP has risen above its CMC, though DEHP- is still far below its $\mathrm{CMC}$, likely making HDEHP the dominant contribution to the reduction in surface tension to $54.1 \mathrm{mN} / \mathrm{m}$. At $\mathrm{pH} 2.0$, without lanthanide ions, the surface tension is further reduced to $43.8 \mathrm{mN} / \mathrm{m}$. In the absence of ions, the reduction in surface tension with $\mathrm{pH}$ follows the increase in concentration of the protonated species, HDEHP, which is expected to be the predominant species at the interface at these values of $\mathrm{pH}$ in the absence of $\mathrm{Ln}$ ions.

pH Dependence with Ln Ions. The surface tension measurements shown in Fig. 2 suggests that (H)DEHP adsorption in the absence of ions is strongest at $\mathrm{pH} 2.0$; yet, when $\mathrm{Nd}$ or Er ions are present in solution, their adsorption is weakest at this $\mathrm{pH}$. This is demonstrated by XFNTR measurements shown in Fig. 4 that measured the area per ion at the interface to be $4,700 \AA^{2}$ for Nd ions to nearly $20,000 \AA^{2}$ for Er ions. Under these low pH conditions, the nearly fully protonated interfacial monolayer is resistant to adsorption of either Nd or Er ions or (H)DEHP complexes containing them. At pH 2.0 the measured surface tension value in the presence of $\mathrm{Nd}$ ions is slightly higher than the value without ions and is much higher in the presence of Er ions (Fig. 2D). Both results suggest that the amount of interfacial (H)DEHP is reduced in the presence of ions, though the resolution of the $\mathrm{x}$-ray reflectivity measurements is not sufficient to confirm this.

At the intermediate $\mathrm{pH}$ 3.0, XFNTR measurements shown in Fig. 4A reveal substantial adsorption of Nd ions, but small to negligible adsorption of Er ions (Table 2). This is consistent with the increase in electron density measured by x-ray reflectivity for Nd-containing samples, either single-Ln-component or mixed Nd+Er ion solutions (Fig. 3E). At this pH, the surface tension for single ionic component Nd solutions is nearly the same as in the absence of ions (Fig. 2D), perhaps suggesting an adsorption of Nd-(H)DEHP complexes at the interface whose interfacial density is similar to that of (H)DEHP in the absence of Ln ions. Surface tensions of solutions containing Er ions are higher than for solutions without ions, again suggesting the reduction in the amount of interfacial (H)DEHP in the presence of Er ions.

Although (H)DEHP adsorption in the absence of Ln ions appears to be low at $\mathrm{pH} 4.5$, ion adsorption is greatest at this $\mathrm{pH}$, as demonstrated by XFNTR measurements in Fig. 4 that measure the area per ion at the interface to be roughly $240 \AA^{2}$ (Table 2). A substantial adsorption at the surface of solutions with $\mathrm{Nd}$ ions is consistent with the reduction in surface tension by $10.3 \mathrm{mN} / \mathrm{m}$ below the value for HDEHP solutions without $\mathrm{Ln}$ ions. A substantial adsorption is also observed when Er ions are in the solution, even though its equilibrium tension value is essentially unchanged from the value without $\mathrm{Ln}$ ions. The very long equilibration time for $\mathrm{Er}$ at this $\mathrm{pH}$, in which the surface tension remains close to the value for pure water (Fig. 2C) and XFNTR measurements reveal only a small adsorption of Er ions for roughly 100 minutes (Fig. S6), suggests that complexation in the bulk aqueous phase may play an important role.

Complexation in the Bulk Aqueous Solution. To address the question of preferential adsorption of light over heavy lanthanide ions to the surface of aqueous solutions of HDEHP, we suggest that different Ln ion-extractant complexes can form within the bulk aqueous phase, where the complexes formed by lighter lanthanides are more hydrophobic and simpler in composition than the complexes formed by heavier lanthanides. We offer this suggestion as an avenue for future research but discuss its consequences for the results presented here. If Ln ion-extractant complexes formed by lighter lanthanides were more hydrophobic (or, equivalently, less hydrophilic) than those formed by heavier lanthanides, the lighter lanthanides would preferentially go to the liquid-vapor interface as observed in our surface tension and XFNTR measurements. 
It is reasonable to expect that lighter and heavier lanthanides will interact differently with (H)DEHP in the bulk aqueous phase as a result of the lanthanide contraction, which expresses the fact that heavier lanthanides have smaller radii. For example, the Er(III) ionic radius is smaller than Nd(III) by $11 \mathrm{pm} .{ }^{48}$ Therefore, Er(III) has a higher effective charge than $\mathrm{Nd}(\mathrm{III})$, that is, while they have the same formal charge (+3), the smaller Er ion has a larger electric field which interacts more strongly with charged DEHP-. The stronger interaction in aqueous solution is demonstrated in Fig. 5 by a molecular dynamics (MD) simulation of the potential of mean force (or free energy profile) of each ion interacting separately with DEHP- in bulk aqueous solution. The free energy minimum is lower for the Er-DEHP- complex by $2.2 \mathrm{kcal} / \mathrm{mol}$ and the equilibrium distance of the ion to the phosphorus atom in DEHP- is slightly smaller for Er(III). At pH 3.0 and pH 4.5, where DEHP- is the dominant species in the bulk aqueous phase, this stronger interaction is expected to lead to greater complexation of DEHP- with Er(III) in the bulk solution. This stronger interaction with Er may form a more stable bulk complex that also has the effect of stabilizing $(\mathrm{H})$ DEHP in the bulk aqueous phase. This is consistent with our surface tension measurements that suggests that the presence of Er can reduce the amount of interfacial (H)DEHP, most likely by stabilizing it in the bulk.

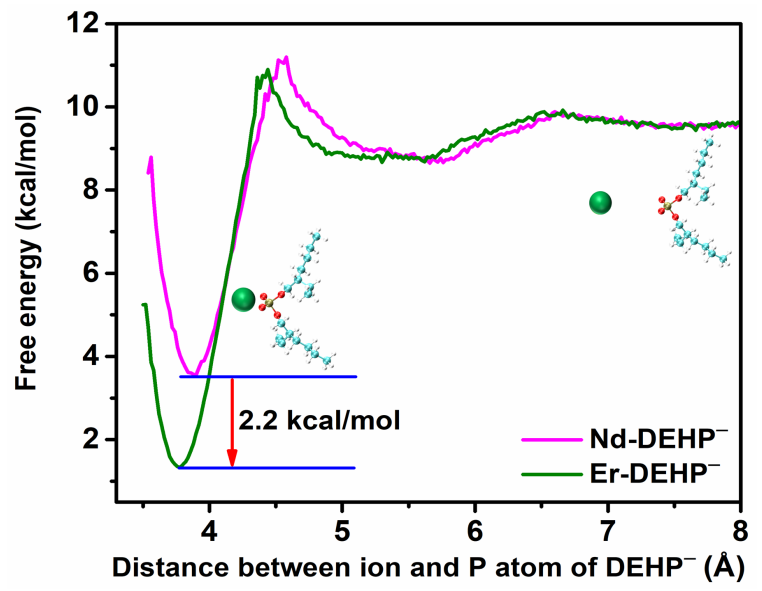

Figure 5. MD simulations of the potential of mean force (PMF or free energy profile) between a single Ln(III) ion and a single DEHPin bulk water (containing two $\mathrm{Cl}^{-}$for electrical neutrality positioned far from the two interacting species), where Ln(III) is either Er(III) or Nd(III). The deeper minimum and slightly smaller ion to P distance for Er(III) reveal a stronger interaction with Er(III) than with Nd(III). The molecular cartoon illustrates Ln(III) in green at a given distances from DEHP- (cyan balls represent carbons, red oxygen, white hydrogen, bronze phosphorus). See SI Section 2 for MD methods.

One form that this bulk complexation might take is illustrated in Fig. 6, which shows an MD simulation of the formation of micelles of DEHP- and Ln ions. These micelles have a roughly 3:1 ratio of DEHP-:Er(III) with the Ln ions on the outside surface of the micelle. Similar micelles were observed for complexes with Nd(III). Additional MD simulations are required to establish the equilibrium state of such bulk complexes and whether or not that equilibrium state is different for light and heavy lanthanides.

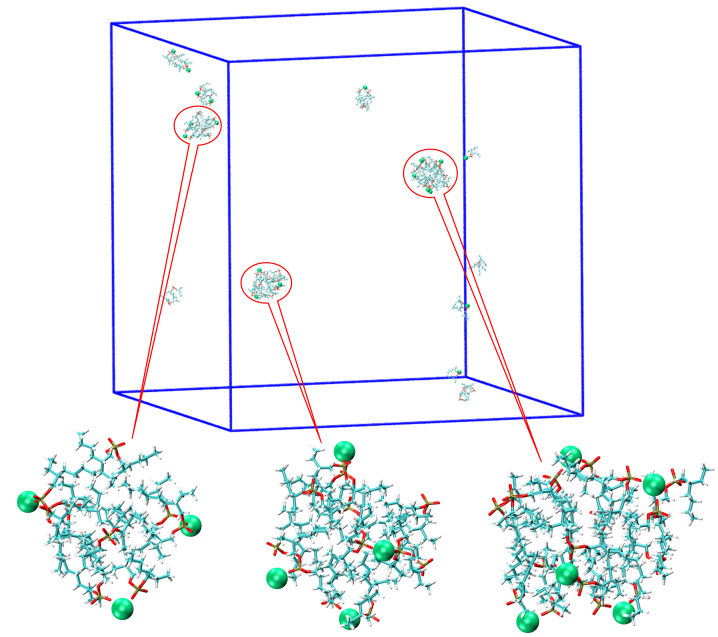

Figure 6. MD simulation of bulk water (without an interface) that illustrates a selection of differently sized micelles formed spontaneously in a bulk aqueous solution of (H)DEHP represented as a stick structure and Er(III) ions represented by green dots (cyan sticks represent carbons, red oxygen, white hydrogen, bronze phosphorus, and the water is hidden). The three micelles shown contain 9 DEHP-:3 Er(III), 12 DEHP-:4 Er(III), and 16 DEHP-:5 Er(III). See SI section 3 for MD methods. 
As mentioned, equilibrium complexes that were more hydrophobic (or less hydrophilic) for light Ln than for heavy Ln could explain the relative preference of light Ln for the surface of aqueous HDEHP solutions. The different kinetics of relaxation of the surface tension shown in Fig. 2 for light and heavy lanthanides could also be explained by a difference in the bulk complexes. If bulk complexes of light lanthanides with (H)DEHP could be easily accommodated at the liquidvapor interface, then it might be expected that the kinetics of adsorption would be similar to the fast adsorption kinetics observed for solutions of HDEHP without lanthanides. These complexes might consist of a relatively small number of components, light Ln-ions and extractants. Conversely, if Ln ion-extractant complexes for heavier lanthanides were more hydrophilic, they would be stabilized in the bulk aqueous phase. Likewise, if these complexes consisted of a larger number of components, e.g., the micelles shown in Fig. 6, which require an additional process to break them into smaller moieties to adsorb to the surface, then the kinetics of adsorption would be longer for heavier lanthanides than for lighter lanthanides. Note that it is unlikely that the large micelles shown in Fig. 6 can adsorb and be stabilized at the liquid-vapor interface to a significant extent because our x-ray reflectivity measurements did not detect them at the interface.

The $\mathrm{pH}$ variation of the relative values of equilibrium surface tension shown in Fig. 2D can also be explained by our suggestion of more hydrophobic, simpler bulk complexes for lighter lanthanides. Figure 2D shows that at $\mathrm{pH} 4.5$, where most of the (H)DEHP species in bulk solution are hydrophilic (charged DEHP-), heavier lanthanides do not greatly change the surface tension from the value without Ln ions, but lighter lanthanides lower the surface tension significantly. If lighter lanthanides form Ln ion-extractant complexes that are more hydrophobic, then these complexes will go to the surface and reduce the surface tension. The more hydrophilic Ln ion-extractant complexes formed by heavier lanthanides will preferentially stay in the bulk, as will the hydrophilic DEHP- for solutions without Ln ions; therefore, the surface tension will be high. At pH 2.0, most of the (H)DEHP species in bulk solution are hydrophobic (uncharged HDEHP) in the absence of Ln ions. Hydrophobic ion-extractant complexes formed by light lanthanides will not change the tension much in comparison to solutions without Ln ions. However, at pH 2.0, the stronger interactions of heavy lanthanides with (H)DEHP can stabilize (H)DEHP in the bulk phase and raise the surface tension.

Ln Ion Adsorption to Water-Insoluble DHDP. If Er(III) has a stronger interaction with DEHP- than does Nd(III), then it might be expected that Er ions would also dominate the interfacial adsorption of (H)DEHP solutions, though this is not observed. We suggested above that the preferential adsorption of light lanthanides is the result of the stronger complexation of heavier Ln ions with (H)DEHP in the bulk aqueous phase. Here, we test this idea by eliminating the bulk complexation by measuring the competitive binding of Nd and Er ions to an insoluble monolayer of di-hexadecyl phosphate (DHDP in Chart 1) at the liquid-vapor interface. DHDP has the same phosphoric acid headgroup as HDEHP, but longer unbranched hydrocarbon tailgroups, which make it insoluble in the aqueous phase and, therefore, confine it to the interface. The initial ratio of ions in bulk aqueous solution was $1: 1(\mathrm{Nd}: \mathrm{Er}$ ) at a concentration of $10 \mu \mathrm{M}$ and a pH of 2.5 . The measurement shown in Fig. 7 illustrates the temporal evolution of ion adsorption to the DHDP monolayer. The first XFNTR measurement, taken 30 minutes after spreading the monolayer, illustrates similar adsorption of both ions to the DHDP monolayer, but soon after Er ion adsorption dominates the interface. A related observation has been reported of preferential adsorption of Er over Nd to an insoluble octadecylphosphonic acid monolayer supported on the surface of an aqueous electrolyte solution. ${ }^{29}$ Here, we have seen that when the phosphoric acid headgroups of water-insoluble DHDP are confined to the liquid-vapor interface, Er ions are preferentially adsorbed, but when water-soluble HDEHP is used, the phosphoric acid headgroups are also in the bulk aqueous solution and Nd ions are preferentially adsorbed to the liquid-vapor interface.

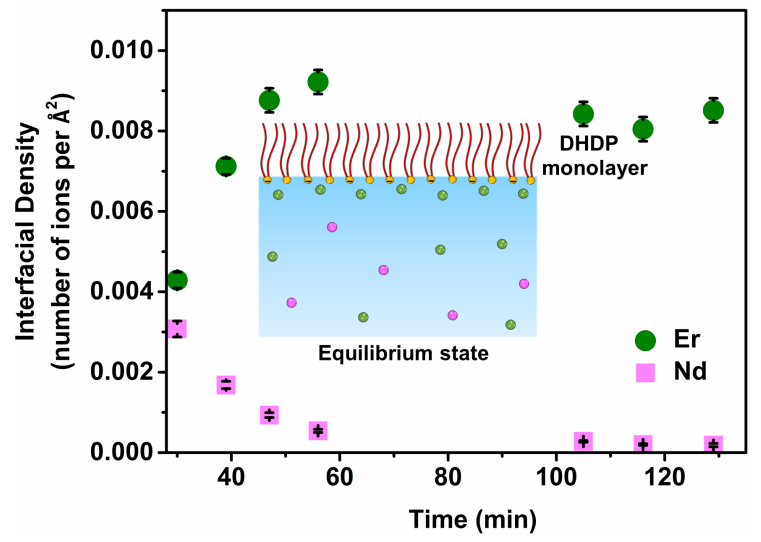

Figure 7. Interfacial density of Er(III) and $\mathrm{Nd}(\mathrm{III})$ at the surface of $\mathrm{pH} 2.5$ aqueous solution supporting an insoluble monolayer of DHDP. These results from XFNTR measurements illustrate the preferential binding of Er ions over Nd ions for the phosphoric acid headgroup of DHDP confined to the interface (see Fig. S7 for XFNTR data and Table S3 for numerical values). 
Implications for Solvent Extraction of Lanthanides by HDEHP. The extractant HDEHP is commonly used in rare earth processes and preferentially extracts heavy over light lanthanides. ${ }^{8}$ In this process, a multi-component aqueous solution of lanthanides is placed in contact with an immiscible organic solvent containing HDEHP. It is believed that ions complex with (H)DEHP near the interface as the result of one or both of the following processes: (Path 1 in Fig. 1) weakly amphiphilic (H)DEHP adsorbs to the liquid-liquid interface, where it then interacts with ions approaching from the waterside of the interface, ${ }^{12}$ or (Path 2 in Fig. 1) HDEHP dissolves in the aqueous phase boundary layer where it complexes with ions, then the ion-extractant complex diffuses back into the organic solvent. ${ }^{15}$ Here, we have shown that HDEHP solvation in the aqueous phase, which might occur in the boundary layer or in the bulk, will be antagonistic to the intended preferential extraction of heavy over light lanthanides for several reasons. Preferential solvation of heavier Lnextractant complexes in the aqueous solution opposes the use of HDEHP to preferentially extract heavier lanthanides into the organic solvent. Contributing to this antagonistic behavior is the preference of light lanthanides for the liquidvapor interface of HDEHP solutions. Also, once complexed with (H)DEHP in bulk aqueous solution, the kinetics of heavier lanthanide adsorption to the interface is longer by several to hundreds of minutes than for lighter lanthanides.

These results raise several issues for the mechanism of liquid-liquid extraction of lanthanides with HDEHP that require further study. However, these results might be relevant only under conditions when the aqueous solution contains more (H)DEHP than ions, as in these experiments which had a 6:1 ratio of (H)DEHP to each ion. Under typical initial conditions for liquid-liquid extraction, the concentration of Ln ions greatly exceeds the concentration of (H)DEHP in the aqueous phase. Therefore, there will not be enough (H)DEHP in the aqueous phase to complex with all Ln ions, which are then available to go to the liquid-liquid interface to be extracted. As liquid-liquid extraction proceeds, equilibration between the two phases will maintain the saturation concentration of (H)DEHP in the aqueous phase, yet the number of heavy Ln ions in aqueous solution will fall faster than the number of light $\mathrm{Ln}$ ions as they are preferentially extracted into the bulk organic phase. Eventually, the amount of (H)DEHP in aqueous solution may exceed that of heavy Ln ions, but not that of the lighter Ln ions. Under these conditions, the antagonistic effects due to complexation in the bulk aqueous phase will limit the extraction of heavy Ln ions. In addition, the preference of lighter Ln ions for the water surface, as revealed in this paper, may enhance their extraction. Both of these effects are antagonistic to the intended preferential extraction of heavier lanthanides by HDEHP.

\section{CONCLUSION}

Measurements of the liquid-vapor interface of aqueous solutions containing a water-soluble extractant, HDEHP, and trivalent lanthanide ions demonstrated the preferential adsorption of light over heavy lanthanides for solution $\mathrm{pH}$ of 2.0, 3.0, and 4.5. Surface tension measurements revealed a sequential variation along the lanthanide series, as well as slower kinetics of adsorption for heavier lanthanides. X-ray reflectivity and x-ray fluorescence near total reflection measurements directly probed the interfacial adsorption of a light lanthanide ion, $\mathrm{Nd}(\mathrm{III})$, and a heavy lanthanide ion, $\operatorname{Er}(\mathrm{III})$, including element-specific measurements of the interfacial density of these ions. These measurements demonstrated the preferential adsorption of Nd over Er ions at the surface of aqueous solutions containing single Ln ionic components, but also revealed the nearly exclusive presence of $\mathrm{Nd}$ at the surface under conditions of competitive adsorption in an aqueous solution containing both types of ions. Our hypothesis that light lanthanides form ion-extractant complexes that are more hydrophobic and simpler in composition than those formed by heavy lanthanides appears to explain the observed differences in adsorption of light and heavy lanthanide ions to the surface of aqueous solutions of (H)DEHP.

In contrast to these measurements with a soluble organophosphoric acid extractant, HDEHP, measurements of interfacial adsorption to a monolayer of a water-insoluble organophosphoric acid extractant, DHDP, which contains the same headgroup but is confined to the interface, demonstrated the inverse preferential adsorption, that is, a preferential adsorption of Er over Nd. This exhibits the same preference for heavy over light lanthanides as utilized by liquid-liquid extraction of these elements by HDEHP. Our results suggest that the presence of water-soluble extractant HDEHP will bias the extraction towards heavier lanthanides when it is located at the aqueous-organic interface, but once HDEHP is solvated in the aqueous solution it becomes antagonistic to the preferential extraction of heavy over light lanthanides. The effects investigated will likely reduce the separation factor between heavy and light lanthanides, thereby leaving excess heavy lanthanide in the aqueous raffinate at the end of the extraction process and complicating the environmental impact of the extraction. Comparative studies at the liquid-liquid interface will be necessary to confirm this suggestion. Our results clarify the role of the aqueous phase and possible advantages of extractants that interact with ions only at the interface during the process of liquid-liquid extraction. These results may have broader applicability in chemical separations because solvent extraction of solutes from an aqueous phase to an organic phase is a separations technique widely used in chemistry, not just for the separation of rare earth elements, and extractants with some solubility in water are not unusual. ${ }^{49}$ 


\section{ASSOCIATED CONTENT}

\section{Supporting Information}

The Supporting Information is available free of charge on the ACS Publications website.

One pdf file containing 7 figures and 3 tables: experimental details - materials, solution preparation; experimental methodology surface tension (including equilibrium values and CMC measurements), $\mathrm{x}$-ray instrumentation and Langmuir trough, $\mathrm{x}$-ray reflectivity (including zero-roughness profiles), XFNTR (including spectral analysis, calibration measurements, interfacial density values, time-dependent data); MD simulation methods.

\section{AUTHOR INFORMATION}

\section{Corresponding Author}

schloss@uic.edu, bu@cars.uchicago.edu.

\section{Present Addresses}

†Zhu Liang, Center for Functional Nanomaterials, Brookhaven National Laboratory, Upton, NY 11973 U.S.A.

\section{Author Contributions}

The manuscript was written through contributions of all authors. All authors have given approval to the final version of the manuscript.

\section{Notes}

The authors declare no competing financial interest.

\section{ACKNOWLEDGMENT}

This research is performed using funding received from the DOE Office of Nuclear Energy's Nuclear Energy University Program under Award Number DE-NE0008779 to MLS, AG, and MAB. This material is also based upon work supported by the U.S. Department of Energy, Office of Science, Office of Basic Energy Sciences Separations Program under Award Number DE-SC0018200 to MLS and IB. NSF's ChemMatCARS Sector 15 is funded by the Divisions of Chemistry (CHE) and Materials Research (DMR), National Science Foundation, under grant number NSF/CHE-1834750. Use of the Advanced Photon Source, an Office of Science User Facility operated for the U.S. Department of Energy (DOE) Office of Science by Argonne National Laboratory, was supported by the U.S. DOE under Contract No. DE-AC02-06CH11357. We thank Frederick Richard for assistance in measuring the x-ray data from pH 2.0 samples.

\section{ABBREVIATIONS}

HDEHP, bis(2-ethylhexyl) phosphoric acid; DHDP, di-hexadecyl phosphoric acid; XFNTR, x-ray fluorescence near total reflection.

\section{REFERENCES}

1. Tasker, P. A.; Plieger, P. G.; West, L. C., Metal Complexes for Hydrometallurgy and Extraction. In Comprehensive Coordination Chemistry II: From Biology to Nanotechnology, McCleverty, J. A.; Meyer, T. J., Eds. Elsevier: Oxford, 2004; Vol. 9, pp 759-808.

2. Huang, X.; Dong, J.; Wang, L.; Feng, Z.; Xuea, Q.; Menga, X., Selective recovery of rare earth elements from ion-adsorption rare earth element ores by stepwise extraction with HEH(EHP) and HDEHP. Green Chemistry 2017, 19, 1345-1352.

3. Cheisson, T.; Schelter, E. J., Rare earth elements: Mendeleev's bane, modern marvels. Science 2019, 363, 489-493.

4. Martinez-Gomez, N. C.; Vu, H. N.; Skovran, E., Lanthanide chemistry: from coordination in chemical complexes shaping our technology to coordination in enzymes shaping bacterial metabolism. Inorganic Chemistry 2016, 55, 10083-10089.

5. $\quad$ Bauer, D.; Diamond, D.; Li, J.; McKittrick, M.; Sandalow, D.; Telleen, P. Critical Minerals Strategy; U.S. Department of Energy 2011; pp 1-189.

6. Peppard, D. F.; Mason, G. W.; Maier, J. L.; Driscoll, W. J., Fractional extraction of the lanthanides as their di-alkyl orthophosphates. Journal of Inorganic and Nuclear Chemistry 1957, 4 (5), 334-343.

7. Sato, T., Liquid-Liquid Extraction of Rare-Earth Elements from Aqueous Acid Solutions by Acid Organophosphorus Compounds. Hydrometallurgy 1989, 22, 121-140.

8. Xie, F.; Zhang, T. A.; Dreisinger, D.; Doyle, F., A critical review on solvent extraction of rare earths from aqueous solutions. Minerals Engineering 2014, 56, 10-28.

9. Huang, X. W.; Long, Z. Q.; Wang, L. S., Technology development for rare earth cleaner hydrometallurgy. Rare Metals 2015, 34, 215222.

10. Watarai, H.; Freiser, H., Role of the Interface in the Extraction Kinetics of Zinc and Nickel Ions with Alkyl-Substituted Dithizones. J Am Chem Soc 1983, 105, 189-190.

11. Gaonkar, A. G.; Neuman, R. D., Interfacial activity, extractant selectivity, and reversed micellization in hydrometallurgical liquidliquid-extraction systems. J. Coll. Int. Sci. 1987, 119 (1), 251-261.

12. Szymanowski, J., Kinetics and interfacial phenomena. Solv. Ex. Ion Ex. 2000, 18, 729-751.

13. Testard, F.; Berthon, L.; Zemb, T., Liquid-liquid extraction: An adsorption isotherm at divided interface? C. R. Chimie 2007, 10, 1034-1041.

14. Prochaska, K., Interfacial activity of metal ion extractant. Adv Colloid Interfac 2002, 95 (1), 51-72. 
15. Hughes, M. A.; Rod, V., A general model to account for the liquid/liquid kinetics of extraction of metals by organic acids. Faraday Discus. Chem. Soc. 1984, 77, 75-84.

16. Dreisinger, D. B.; Cooper, W. C., The kinetics of cobalt and nickel extraction using HEHEHP. Solv. Extract. Ion Exch. 1986, 4 (2), 317344.

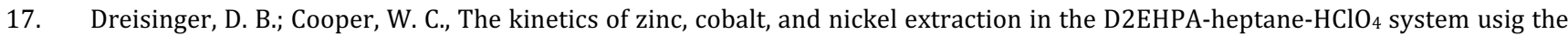
rotating diffusion cell technique. Solv. Extract. Ion Exch. 1989, 7 (2), 335-360.

18. Honaker, C. B.; Freiser, H., Kinetics of Extraction of Zinc Dithizonate. The Journal of Physical Chemistry 1962, 66 (1), $127-130$.

19. Bu, W.; Schlossman, M. L., X-ray Studies of Liquid Interfaces in Model Solvent Extraction Systems. In Ion Exchange and Solvent Extraction: Volume 23 Changing the Landscape in Solvent Extraction, Moyer, B. A., Ed. CRC Press, Taylor \& Francis Group: Boca Raton, London, New York, 2019; pp 115-146.

20. Bu, W.; Hou, B.; Mihaylov, M.; Kuzmenko, I.; Lin, B.; Meron, M.; Soderholm, L.; Luo, G.; Schlossman, M. L., X-ray fluorescence from a model liquid/liquid solvent extraction system. J. Appl. Phys. 2011, 110, 102214-1 - 102214-6.

21. Bu, W.; Yu, H.; Luo, G.; Bera, M. K.; Hou, B.; Schuman, A. W.; Lin, B.; Meron, M.; Kuzmenko, I.; Antonio, M. R.; Soderholm, L.; Schlossman, M. L., Observation of a rare earth ion-extractant complex arrested at the oil-water interface during solvent extraction. J. Phys. Chem. B 2014, 118, 10662-10674.

22. Liang, Z.; Bu, W.; Schweighofer, K. J.; Jr., D. J. W.; Harvey, J. S.; Hanlon, G. R.; Amoanu, D.; Erol, C.; Benjamin, I.; Schlossman, M. L., A nanoscale view of assisted ion transport across the liquid-liquid interface. Proc. Nat. Acad. Sci. (USA) 2019, 116, 18227-18232.

23. Scoppola, E.; Watkins, E.; Destri, G. L.; Porcar, L.; Campbell, R. A.; Konovalov, O.; Fragnetoa, G.; Diat, O., Structure of a liquid/liquid interface during solvent extraction combining X-ray and neutron reflectivity measurements. Phys. Chem. Chem. Phys. 2015, 17, 15093-15097.

24. Scoppola, E.; Watkins, E. B.; Campbell, R. A.; Konovalov, O.; Luc Girard, J.-F. D.; Ferru, G.; Fragneto, G.; Diat, O., Solvent extraction: Structure of the liquid-liquid interface containing a diamide ligand. Angew. Chem., Int. Ed. 2016, 128, 9472 -9476.

25. Chowdhury, A. U.; Lin, L.; Doughty, B., Hydrogen-Bond-Driven Chemical Separations: Elucidating the Interfacial Steps of SelfAssembly in Solvent Extraction. ACS Applied Materials \& Interfaces 2020, 12 (28), 32119-32130.

26. Wang, W. J.; Park, R. Y.; Meyer, D. H.; Travesset, A.; Vaknin, D., Ionic Specificity in pH Regulated Charged Interfaces: Fe ${ }^{3+}$ versus $\mathrm{La}^{3+}$. Langmuir 2011, 27 (19), 11917-11924.

27. Sung, W.; Vaknin, D.; Kim, D., Different Adsorption Behavior of Rare Earth and Metallic Ion Complexes on Langmuir Monolayers

Probed by Sum-Frequency Generation Spectroscopy. Journal of the Optical Society of Korea 2013, 17, 10-15.

28. Miller, M.; Chu, M.; Lin, B.; Bu, W.; Dutta, P., Atomic Number Dependent "Structural Transitions" in Ordered Lanthanide Monolayers: Role of the Hydration Shell. Langmuir 2017, 33 (6), 1412-1418.

29. Miller, M.; Liang, Y.; Li, H.; Chu, M.; Yoo, S.; Bu, W.; Olvera de la Cruz, M.; Dutta, P., Electrostatic Origin of Element Selectivity during Rare Earth Adsorption. Physical Review Letters 2019, 122 (5), 058001.

30. Rock, W.; Qiao, B.; Zhou, T.; Clark, A. E.; Uysal, A., Heavy Anionic Complex Creates a Unique Water Structure at a Soft Charged Interface. The Journal of Physical Chemistry C 2018, 122 (51), 29228-29236.

31. Uysal, A.; Rock, W.; Qiao, B.; Bu, W.; Lin, B., Two-Step Adsorption of PtCl62- Complexes at a Charged Langmuir Monolayer: Role of Hydration and Ion Correlations. The Journal of Physical Chemistry C 2017, 121 (45), 25377-25383.

32. Neal, J. F.; Zhao, W.; Grooms, A. J.; Smeltzer, M. A.; Shook, B. M.; Flood, A. H.; Allen, H. C., Interfacial Supramolecular Structures of Amphiphilic Receptors Drive Aqueous Phosphate Recognition. J Am Chem Soc 2019, 141 (19), 7876-7886.

33. Nayak, S.; Lovering, K.; Bu, W.; Uysal, A., Anions Enhance Rare Earth Adsorption at Negatively Charged Surfaces. The Journal of Physical Chemistry Letters 2020, 11 (11), 4436-4442.

34. Lovering, K.; Nayak, S.; Bu, W.; Uysal, A., The Role of Specific Ion Effects in Ion Transport: The Case of Nitrate and Thiocyanate. The Journal of Physical Chemistry C 2020, 124 (1), 573-581.

35. Grooms, A. J.; Neal, J. F.; Ng, K. C.; Zhao, W.; Flood, A. H.; Allen, H. C., Thermodynamic Signatures of the Origin of Anti-Hofmeister Selectivity for Phosphate at Aqueous Interfaces. The Journal of Physical Chemistry A 2020, 124 (27), 5621-5630.

36. Neal, J. F.; Saha, A.; Zerkle, M. M.; Zhao, W.; Rogers, M. M.; Flood, A. H.; Allen, H. C., Molecular Recognition and Hydration Energy Mismatch Combine To Inform Ion Binding Selectivity at Aqueous Interfaces. The Journal of Physical Chemistry A 2020, 124 (49), 1017110180.

37. Nayak, S.; Kumal, R. R.; Liu, Z.; Qiao, B.; Clark, A. E.; Uysal, A., Origins of Clustering of Metalate-Extractant Complexes in LiquidLiquid Extraction. ACS Applied Materials \& Interfaces 2021, 13 (20), 24194-24206.

38. Kusaka, R.; Watanabe, M., The structure of a lanthanide complex at an extractant/water interface studied using heterodynedetected vibrational sum frequency generation. Phys Chem Chem Phys 2018, 20 (4), 2809-2813.

39. Kusaka, R.; Watanabe, M., Stoichiometry of Lanthanide-Phosphate Complexes at the Water Surface Studied Using Vibrational Sum Frequency Generation Spectroscopy and DFT Calculations. J. Phys. Chem. B 2021, 125 (24), 6727-6731.

40. Rama Swami, K.; Kumaresan, R.; Nayak, P. K.; Venkatesan, K. A.; Antony, M. P., Effect of pKa on the extraction behavior of Am(III) in organo phosphorus acid and diglycolamide solvent system. Radiochimica Acta 2018, 106 (2), 107-118.

41. Welbourn, R. J. L.; Lee, S. Y.; Gutfreund, P.; Hughes, A.; Zarbakhsh, A.; Clarke, S. M., Neutron Reflection Study of the Adsorption of the Phosphate

Surfactant NaDEHP onto Alumina from Water. Langmuir 2015, 31, 3377-3384.

42. Schlossman, M. L.; Synal, D.; Guan, Y.; Meron, M.; Shea-McCarthy, G.; Huang, Z.; Acero, A.; Williams, S. M.; Rice, S. A.; Viccaro, P. J., A synchrotron X-ray liquid surface spectrometer. Rev. Sci. Instrum. 1997, 68, 4372-4384.

43. Lin, B.; Meron, M.; Gebhardt, J.; Graber, T.; Schlossman, M. L.; Viccaro, P. J., The liquid surface/interface spectrometer at ChemMatCARS synchrotron facility at the Advanced Photon Source. Physica B 2003, 336, 75-80.

44. Launiere, C. A.; Gelis, A. V., High Precision Droplet-Based Microfluidic Determination of Americium(III) and Lanthanide(III) Solvent Extraction Separation Kinetics. Ind Eng Chem Res 2016, 55 (7), 2272-2276.

45. Nichols, K. P.; Pompano, R. R.; Li, L.; Gelis, A. V.; Ismagilov, R. F., Toward Mechanistic Understanding of Nuclear Reprocessing Chemistries by Quantifying Lanthanide Solvent Extraction Kinetics via Microfluidics with Constant Interfacial Area and Rapid Mixing. J Am Chem Soc 2011, 133 (39), 15721-15729.

46. Pershan, P. S.; Schlossman, M. L., Liquid Surfaces and Interfaces: Synchrotron X-ray Methods. Cambridge University Press: Cambridge, 2012. 
47. Bevington, P. R.; Robinson, D. K., Data Reduction and Error Analysis for the Physical Sciences. 3rd Ed., Ch. 11. McGraw Hill: New York, 2003.

48. Marcus, Y., Ionic Radii in Aqueous Solutions. Chem. Rev. 1988, 88, 1475-1498.

49. Sekine, T.; Hasegawa, Y. k., Solvent Extraction Chemistry: Fundamentals and Applications. M. Dekker: New York, 1977. 
Insert Table of Contents artwork here

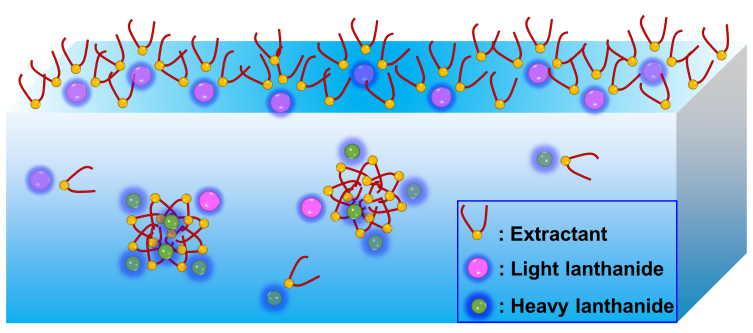

